\title{
Pleural Fluid Analysis: Standstill or a Work in Progress?
}

\author{
T. Hassan, M. Al-Alawi, S. H. Chotirmall, and N. G. McElvaney \\ Respiratory Research Division, Educationand Research Center, Royal College of Surgeons in Ireland, Beaumont Hospital, \\ Beaumont Road, Dublin 9, Ireland \\ Correspondence should be addressed to S. H. Chotirmall, schotirmall@rcsi.ie
}

Received 5 September 2011; Accepted 26 October 2011

Academic Editor: Takashi Nakano

Copyright (c) 2012 T. Hassan et al. This is an open access article distributed under the Creative Commons Attribution License, which permits unrestricted use, distribution, and reproduction in any medium, provided the original work is properly cited.

\begin{abstract}
Pleural fluid analysis yields important diagnostic information in pleural effusions in combination with clinical history, examination, and radiology. For more than 30 years, the initial and most pragmatic step in this process is to determine whether the fluid is a transudate or an exudate. Light's criteria remain the most robust in separating the transudate-exudate classification which dictates further investigations or management. Recent studies have led to the evaluation and implementation of a number of additional fluid analyses that may improve the diagnostic utility of this method. This paper discusses the current practice and future direction of pleural fluid analysis in determining the aetiology of a pleural effusion. While this has been performed for a few decades, a number of other pleural characteristics are becoming available suggesting that this diagnostic tool is indeed a work in progress.
\end{abstract}

\section{Introduction}

Pleural effusions are associated with a number of medical conditions causing fluid accumulation via differing yet synergistic mechanisms including increased pleural membrane permeability, increased pulmonary capillary pressure, decreased oncotic pleural pressure, and lymphatic obstruction. Pleural fluid analysis yields important diagnostic information in most cases of pleural effusions. Standard workup includes determining whether the effusion is transudative or exudative, an important differentiation aiding the physician in narrowing the differential diagnosis (Figure 1). Despite this, several experts propose that such a categorical division represents outdated practice as it does not permit establishing a definitive cause of the effusion. A variety of nonroutine tests may be performed during pleural fluid analysis either as lone or additional diagnostic tools to further determine a definitive cause for an effusion in the appropriate setting. This paper will discuss the current practice and future prospective direction for the use of pleural fluid analysis in determining the aetiology of a pleural effusion in a variety of clinical settings.

\section{Have We Moved on from Light's Criteria?}

The primary aim when investigating a pleural effusion is to establish the correct diagnosis with minimal investigation. Prior to the advent of Light's criteria, most physicians initially determined whether an effusion was transudative or exudative based on the pleural protein level [1]. Serum and fluid albumin gradients of greater than $12 \mathrm{~g} / \mathrm{L}$ also indicated exudates, however, when used in isolation, these criteria have low sensitivity [2].

Light's criteria have recommended for use when a pleural protein is between 25 and $35 \mathrm{~g} / \mathrm{L}$ and defines exudative pleural effusions as having either (1) a ratio $>0.5$ between total pleural and plasma protein, (2) a ratio $>0.6$ between pleural and plasma lactate dehydrogenase (LDH), and (3) pleural LDH higher than two thirds of the normal serum level. The sensitivity of Light's criteria in identifying exudative pleural effusions is high (98\%); however, its ability to exclude transudates remains low. For instance, prospective work by Porcel et al. reported an almost $100 \%$ sensitivity for exudates but found that approximately one-fifth of patients with congestive cardiac failure on diuretics also met Light's criteria for an exudate [3]. Despite this deficiency, Light's criteria 


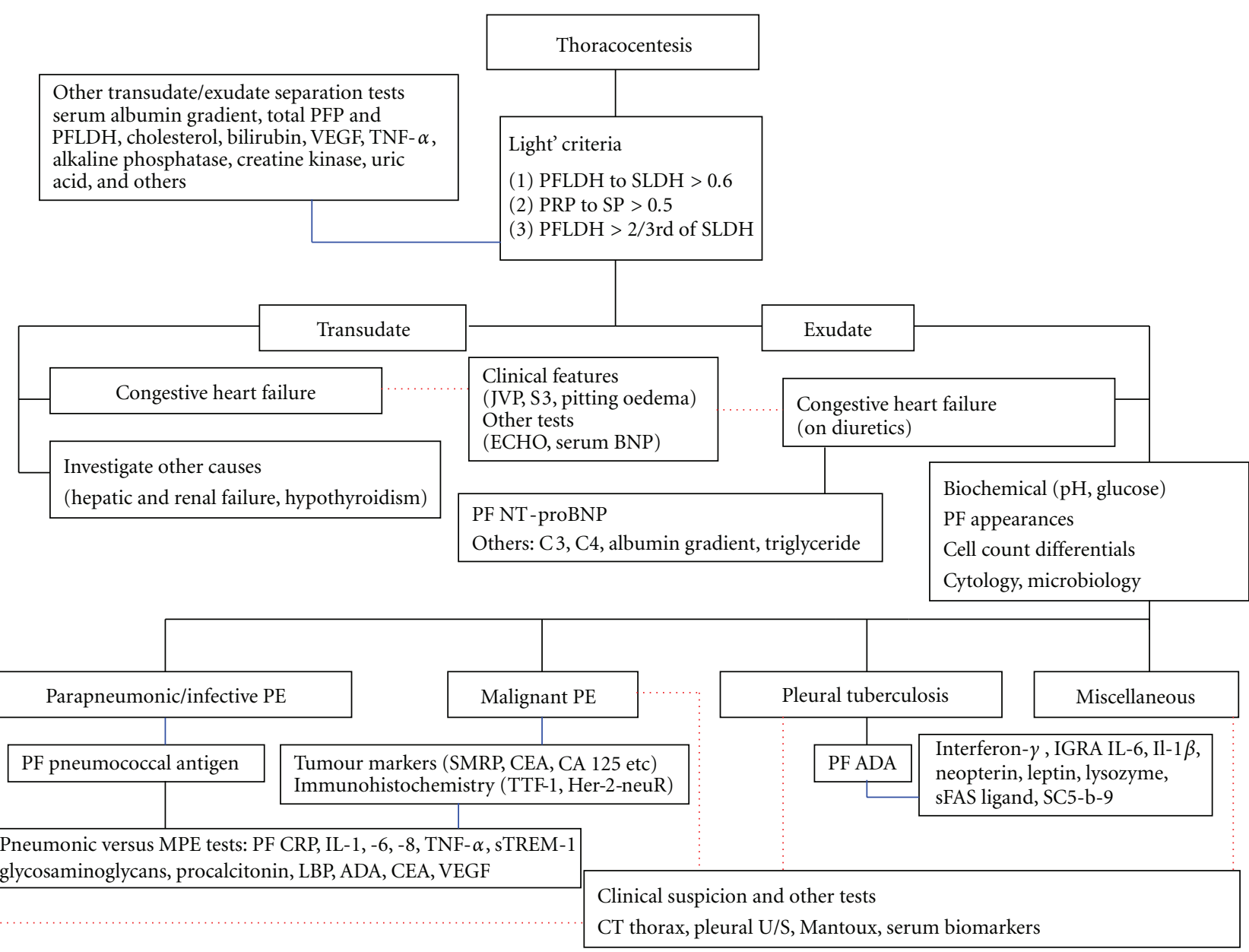

FIgURE 1: Recommended algorithm for investigation of pleural effusion. The use of Light's criteria is recommended when a thoracocentesis revealed a protein level between 25 and $35 \mathrm{~g} / \mathrm{L}$ to narrow down the differential diagnosis by determining whether a pleural effusion is transudative or exudative. NT-proBNP should be measured when a suspected cardiac effusion meets the exudative criteria. Determining causes of an exudative effusion is more challenging, and routine test, including biochemical measurement (i.e., pH and glucose), differential cell counts, cytology, and routine microbiology test are diagnostically useful. Pleural fluid pneumococcal antigen has been shown to be superior than urinary antigen to identify bacterial-induced pleural effusion. Tumour marker such as SMRP has a good diagnostic value to diagnose mesothelioma, however, the diagnostic utility of other tumour markers remains limited. Immunocytochemical evaluation of pleural fluid specimen is helpful in labelling different tumour markers. Other biological markers to differentiate parapneumonic/infective and malignant effusion remain elusive, expensive, and not widely available. Testing of pleural fluid ADA is an inexpensive and efficacious method for diagnosing tuberculous effusion, regardless of the patient's immune status. Other tuberculosis-related inflammatory markers are available but are not superior to the latter. (PF: pleural fluid, black continuous line: strongly recommended and routinely practised, blue continuous line: not strongly recommended and not routinely practised, red dotted line: complementary diagnosis with other nonpleural tests.)

remain superior to clinical judgement for discriminating between transudates and exudates. A prospective study of $n=249$ patients directly comparing clinical suspicion to Light's criteria reported that the former was significantly less accurate to the latter (84\% versus 93\%, $P<0.01)$ (6), illustrating the criteria's importance in routine clinical practice [4].

Most studies to date have focused on making Light's criteria more practical without affecting its discriminatory power. For example, it has been suggested that serum LDH in isolation does not increase the value of the two other criteria components [5]. This is supported by a Brazilian study proposing new criteria to differentiate the two effusion types
[6]. By quantifying exclusively total pleural protein and LDH without the need of serum samplings, this study showed a diagnostic yield comparable to that of Light's criteria.

Other studies have additionally generated a number of nonroutine biochemical measurements on pleural fluid that may discriminate transudates from exudates, for instance pleural fluid bilirubin with pleural : serum ratio of $>0.6$ is suggestive of an exudate with a sensitivity and specificity of $90.6 \%$ and $96.2 \%$, respectively [7]. An early case series has demonstrated that pleural fluid cholesterol $>60 \mathrm{mg} / \mathrm{dL}$ is indicative of an exudative effusion with high sensitivity [8], whilst another study utilizing both LDH and pleural cholesterol measurements revealed similar sensitivity to 
distinguish between the two types of effusion [9]. Vascular endothelial growth factor (VEGF), an important mediator of angiogenesis and vascular permeability, may be another key mediator and thus marker in the identification of exudates due to the increased pleural endothelial permeability it confers. In one study of $n=79$ patients, median levels of VEGF in exudates were approximately twice that of transudates [10]. In addition, elevated tumor necrosis factor alpha (TNF$\alpha$ ) levels are found in both infectious and malignant pleural effusions, but rarely transudates [11]. Other biochemical parameters that have been examined to aid transudate versus exudate differentiation include alkaline phosphatase, creatine kinase, and uric acid which possessed diagnostic accuracies lower than the traditional Light's criteria [12].

Some of these studies have proposed new and alternative criteria avoiding venepuncture consequently reducing investigations and diagnostic costs. However, these criteria vary in their cut-off points to best discriminate the two forms of effusion. To date, Light's criteria remain robust with diagnostic accuracy of $96 \%$ and for now remains the optimal method to separate pleural transudates from exudates.

\section{Cardiac Pleural Effusion: Extending beyond the Transudate/Exudate Boundary}

Cardiac failure remains the most common cause of transudative pleural effusions. A single study has revealed that $28 \%$ of cardiac-related pleural effusions were misclassified as exudative due to the use of diuretics [13]. Brain natriuretic peptide (BNP) is a neuroendocrine hormone secreted from the ventricular walls in response to increased pressures and stretch conferred on cardiomyocytes impacting upon the renin-angiotensin system to increase diuresis and vasodilation [14]. BNP is cleaved to NT-proBNP, and detection of the cleaved product in serum has been used to distinguish cardiac failure from primary pulmonary causes of dyspnoea. This marker has been demonstrated to possess diagnostic usefulness during pleural fluid analysis. A study by Long et al. demonstrated that, although levels of pleural BNP have a statistically significant correlation with NT-proBNP, the latter is a far more accurate diagnostic tool during evaluation of cardiac pleural effusions [15]. In addition to high sensitivity and specificity, pleural NT-proBNP is shown to be superior to Light's criteria for the identification of cardiac-based pleural effusions [16]. Meta-analyses have shown that the number of misclassified effusions from application of Light's criteria was significantly reduced with the use of NT-proBNP [17]. However, there is a need for caution as levels of NTproBNP are physiologically raised in the elderly and renal failure populations requiring further studies to evaluate its role in these groups. Importantly, NT-proBNP measurement is difficult and costly when compared to application of traditional criteria such as the albumin gradient in assessing pleural effusions [18]. Measuring pleural NT-proBNP should therefore be reserved for settings where a suspected cardiac effusion meets exudative criteria but a high index of clinical suspicion remains. Alternative markers have been studied in the assessment of cardiac pleural effusions, for example, normal complement levels (C3 and C4) are reported to have a high negative predictive value in this setting [19].

\section{The Promise of Biological Pleural Markers to Determine the Aetiology of Pleural Exudates}

The most challenging aspect of investigating exudative pleural effusions is differentiating the likelihood of inflammatory parapneumonic versus malignant disease both major causes of exudates in routine clinical practice. Tuberculous effusions are an additional important consideration owing to longterm treatment strategies. Biochemical analyses such as protein, $\mathrm{pH}$, and microbiologic assessment remain the standard investigations during this process. Additionally, cytological examination of suspected malignant pleural effusion (MPE) can result in false-negative rates of up to $40 \%$ [20]. The diagnostic yield for cytology, however, depends on the tumour type; highest for ovarian $(83 \%)$ and less so for breast (78\%), lung $(57 \%)$, and mesothelioma (41\%) primaries. Overall, standard testing to determine the underlying cause of exudates has a suboptimal accuracy requiring other parameters such as clinical suspicion and radiology to play an associative role.

4.1. Pleural Fluid Appearance. Pleural fluid appearance is a nonspecific and undermined tool in the assessment of a pleural effusion. Prior work has suggested that malignancy is the leading cause of gross bloody effusions (47\%) [21] and further confirmed by Porcel and Vives who reported that pleural effusions with significantly higher red blood cell counts occurred in those who subsequently had malignant rather than nonmalignant effusions [22]. In a separate study assessing patients without any prior diagnosis of malignancy, an association between blood-stained effusions and the presence of malignant cells on cytological examination was described [23]. Conversely, a larger retrospective study assessing patients diagnosed with cancer who underwent thoracocentesis revealed no difference between the presence of blood and the ability to predict positive pleural fluid cytology [24]. Therefore, due to low sensitivity and specificity of bloody effusions to indicate malignancy in the setting of an exudate, pleural fluid appearance should not be emphasized as a diagnostic tool to establish MPE as it can be relatively nonspecific.

On the other hand, there are cases where the appearance of pleural fluid may be helpful. The best described setting is one of a milky appearance suggestive of chylothorax or pseudochylothorax that can be differentiated by centrifugation. Although reliable where present, the gross appearance of a chylothorax has been described as nonmilky half the time [25]. Chylothorax may alternatively give the appearance of bile-stained fluid. However, pleural triglyceride analysis is a more definitive test with a level greater than $110 \mathrm{mg} / \mathrm{dL}$ reflecting a $99 \%$ chance that the fluid is chyle. For infectiverelated pleural effusions, an anchovy-brown fluid may indicate amoebic liver abscess whilst black fluid suggests Aspergillus infection [26]. 
4.2. Cell Count Differential. Differential cell count of pleural fluid may also provide clues to the origin of an exudative pleural effusion. Neutrophilic predominance indicates an acute injury of the pleural surface that may occur in parapneumonic settings, pulmonary embolism, and subphrenic abscesses. In chronic, long standing pleural injury, the fluid becomes populated by lymphocytes. Two-thirds of lymphocytic predominant effusions are the result of malignancy or tuberculosis (TB) [27]. Eosinophilic pleural effusions, defined as a pleural effusion that contains at least $10 \%$ eosinophils, most commonly occur during conditions associated with the presence of blood or air in the pleural space such as pneumothorax and malignancy. Interestingly, although eosinophilia is nonspecific and can occur in benign-related effusions, a percentage of pleural eosinophils $>40 \%$ indicates an extremely low likelihood of malignancy [28].

4.3. Markers of Pleural Inflammation. Pleural biological markers have been proposed as an alternative means to determine the cause of exudative pleural effusions. C-reactive protein is an acute-phase reactant widely used as a marker of inflammation and tissue injury. Pleural CRP is found to be higher in benign versus malignant exudates with a sensitivity and specificity of $93.7 \%$ and $76.5 \%$ for parapneumonic effusion $[29,30]$. This finding has been supported by further studies revealing an almost $100 \%$ sensitivity for a cutoff value of $5.3 \mathrm{mg} / \mathrm{dL}$ to identify parapneumonic versus tuberculous or malignant effusions [31]. Interleukin-8 (IL8), a proinflammatory cytokine, and CRP together may differentiate complicated from uncomplicated parapneumonic effusions with sensitivities of $84 \%$ and $72 \%$ and specificities of $82 \%$ and $71 \%$, respectively [32]. Interleukin-6 (IL-6), an alternative proinflammatory cytokine induced by lipopolysaccharide (LPS) as a marker of system activation, has also been shown to effectively differentiate infective from malignant effusions with highest levels in tuberculous rather than parapneumonic effusions [33]. In spite of this, elevated IL- 6 has also been found in MPE, particularly following pleurodesis. Elevated TNF- $\alpha$ is also useful in differentiating tuberculous from malignant effusions [34]. Studies supporting the utility of pleural proinflammatory cytokines during pleural fluid analysis revealed that a low absolute neutrophil count may give a diagnosis of empyema with a sensitivity rate of $78.6 \%$ and a specificity rate of $88.4 \%$ in the presence of elevated IL-8. Similar findings are reported with IL-1 [35, 36].

Other features of pleural fluid analysis that discriminate infection from inflammation in the complicated versus noncomplicated setting include pleural soluble triggering receptor of myeloid cells-1 (sTREM-1), procalcitonin, and lipopolysaccharide binding protein (LBP) [37]. Pleural pneumococcal antigen assays have been explored and may be more sensitive than the equivalent urinary assays for the establishment of microbial-induced pneumonias [38]. Parapneumonic effusions secondary due to S. pneumoniae are further shown to have positive antigen testing in pleural fluid whilst negative results from concurrent urine sampling.
A prospective study of $n=72$ patients set out to discriminate exudates with multiple pleural biological parameters including adenosine deaminase (ADA), CRP, carcinoembryogenic antigen (CEA), IL-6, TNF- $\alpha$, and VEGF found $\mathrm{ADA}$ and CRP to be the most reliable of the group assessed [39]. ADA concentrations $>45 \mathrm{U} / \mathrm{L}$ and $\mathrm{CRP}<4 \mathrm{mg} / \mathrm{dL}$ most likely indicated tuberculous effusions, whilst ADA $<40 \mathrm{U} / \mathrm{L}$ and $\mathrm{CRP}>6 \mathrm{mg} / \mathrm{dL}$ suggested a parapneumonic origin. The latter ADA levels in combination with $\mathrm{CRP}<4 \mathrm{mg} / \mathrm{dL}$ on the other hand, were most likely malignant in origin. In a specialized subgroup of lung transplant recipients, normal or high complement levels within pleural fluid indicate a secondary cause, for example, parapneumonic effusion rather than those attributable to the surgery itself [19].

Despite the large volume of work in-progress, larger and more robust studies are necessitated before we can safely recommend the use of nonroutine and costly biological markers as standard to improve the diagnostic accuracy during the routine workup of an exudative pleural effusion.

4.4. Pleural Tuberculosis. Pleural tuberculosis displays important pleural fluid features that significantly contribute to the diagnostic process. Such features preclude the need for invasive investigation such as thoracoscopy or pleural biopsy. Microbiologic assessment remains paramount directly aiding treatment strategy. Microscopic examination of ZiehlNeelson stained pleural fluid detects acid-fast bacilli in $<5 \%$ of non-HIV cases [40]. Addition of Lowenstein-Jensen media culture increases this positive yield to approximately $35 \%$. Nucleic acid amplification confers better statistics for diagnosis and has specificity between 90\%, and $-97 \%$; however, sensitivity may be as low as 60\% [41].

ADA is a T-cell (CD4+) metalloenzyme whose presence in high levels within pleural fluid strongly indicated tuberculosis particularly in high prevalence areas. High pleural ADA is also detected in non-TB settings including malignancy, rheumatoid arthritis, systemic lupus erythematosis, and parapneumonic effusions. In view of this, it is important to acknowledge that two ADA isoenzymes exist; ADA 1 and 2 with the latter related in increased levels in the tuberculous setting. In meta-analyses of 63 studies, ADA is reported to have a sensitivity of $92 \%$ and a specificity of $90 \%$ [42], whilst within the setting of a lymphocytic predominant effusion, ADA $>40 \mathrm{U} / \mathrm{L}$ is almost exclusively secondary to tuberculosis. Paradoxically, retrospective study of 221 patients has illustrated that ADA levels >250 U/L do not generally occur in tuberculosis related effusions [43]. Such levels are in fact found in patients with empyema or lymphoidrelated malignancies. Therefore, whilst the measurement of pleural fluid ADA remains a useful diagnostic tool for tuberculous pleurisy, it should be interpreted in parallel with clinical findings and other traditional methods such as the tuberculin skin test to reach a diagnosis. Such a combination of clinical features with pleural ADA measurement has excellent diagnostic value with a sensitivity and specificity rates of $95 \%$ and $97 \%$, respectively. Therefore, experts have recommended measuring ADA levels in low-prevalence areas as a concentration $<40 \mathrm{U} / \mathrm{L}$ almost exclusively rules out tuberculosis-driven effusions [44]. 
Measurement of pleural interferon- $\gamma$, an alternative cytokine derived from lymphocytes, may also be utilized in the diagnosis of tuberculous effusions. However, like ADA, elevated levels of interferon- $\gamma$ are reported in empyema and malignancy. Even though the sensitivity and specificity of this marker is lower than that of ADA, joint sensitivity and specificity utilizing both together increases to $96 \%$ and $93 \%$, respectively [45]. A single prospective study of $n=63$ patients illustrated that interferon- $\gamma$ gamma assays (IGRAs) including the commercially available QuantiFERON-TB Gold and T-SPOT-TB performed poorly compared to interferon- $\gamma$ levels $>0.31 \mathrm{IU} / \mathrm{mL}$ as a cut-off value [46]. Use of IGRA is currently not recommended due to variability in results when compared to other markers such as ADA that appear superior.

Other biological parameters including pleural IL-6, IL$1 \beta$, neopterin, leptin, lysozyme, and soluble FAS ligand have extensively been studied in the setting of tuberculous pleural effusions. Neopterin is a pteridine, released by activated macrophages and shown to be elevated in tuberculous effusions [47]. Conversely, leptin, an adipose-derived hormone, has been shown to be reduced to a greater extent in tuberculous effusions when compared to other exudative effusions with a sensitivity and specificity of $82 \%$ [48]. Lysozyme also released from activated macrophages similarly have a sensitivity of $85 \%$ and specificity $61 \%$ for the identification of tuberculous effusions. SC5b-9, a product derived from the binding of C5b-9 complexes to the S protein, is elevated in effusions secondary to TB particularly at a cut-off value of $>2 \mathrm{mg} / \mathrm{L}$. Such measurements have also been studied to aid differentiating tuberculous from malignant pleural effusions [49]. Pleural interferon- $\gamma$ inducible $10 \mathrm{k}-\mathrm{Da}$ protein (IP-10), interleukin-12 p40, and matrix metalloproteinase (MMP) levels [50-52] are additional markers elevated in pleural fluid from tuberculous effusions compared with malignant and other benign settings. Despite this, their significant variability in sensitivity and specificity coupled with costs for routine use preclude the introduction of such measures into routine clinical practice. What is probably most feasible is a combination of these tests used synergistically at a reduced cost. For example, a study has shown that a combination assay including ADA, interferon- $\gamma$, and nucleic acid amplification for TB will have superior sensitivity and specificity as compared to a single test alone and offers a future promise in the workup of tuberculous effusions [53].

4.5. Tumour Markers. Nodularity, pleural and diaphragmatic thickening are highly indicative of malignant pleural disease with a positive predictive value of $100 \%$. Despite this, such features are not always present and pleural fluid cytology thus plays a crucial role in the diagnosis of malignancy. MPEs are positive in $40-60 \%$ of cases [54], and it is common practice to require a large volume $(>500 \mathrm{mLs})$ of fluid to reach a diagnosis by cytology. Interestingly, a recent prospective study on $n=121$ thoracocentesis showed that diagnostic accuracy was dependent on the volume of pleural fluid obtained with a recommendation of $>150 \mathrm{~mL}$, whilst another prospective study examining $n=44$ patients concluded that samples $>50 \mathrm{~mL}$ similarly did not increase diagnostic yields $[55,56]$. Work on noninvasive tumour biomarkers remain ongoing and if successful may avoid invasive investigation such as pleural biopsy or thoracoscopy.

Well-described tumour markers such as carcinoembryonic antigen (CEA), cancer antigen 125 (CA 125), 15-3 (CA 15-3), and neuron-specific enolase/cytokine fragment (CYFRA) 21-1 have limited usefulness in the routine workup of a suspected malignant pleural effusion. CA 125 is a welldescribed tumour marker implicated in ovarian malignancy and has reported high levels in pleural fluid compared to serum in this setting [57]. High pleural CA 125, however, is also observed in squamous and adenocarcinomatous malignant effusions and may have a prognostic role [58]. Pleural CEA was one of the first markers to be evaluated in lung cancer. It is overexpressed in metastatic adenocarcinoma possessing prognostic relevance in terms of median survival and treatment response [59]. Although CEA has been shown to be specific, sensitivity remains low $(<50 \%)$ with variable cut-off values precluding routine use. Metastatic breast cancer is another common cause for malignant pleural effusions with CA 15-3 used for both diagnosis and therapeutic monitoring [60]. CYFRA 21-1, a cytokeratin tumour marker has both diagnostic and prognostic roles in nonsmall cell lung cancer [60]. However, due to relatively poor sensitivity for a single test, use of a combination of tumour markers such as CA 125 and CYFRA 21-1 for adenocarcinoma and CEA, CA 15-3, and CYFRA 21-1 for squamous cell cancer of the lung has been recommended. In a study involving $n=243$ and $n=173$ patients with malignant and benign effusions, respectively, selected cut-off values had to be $100 \%$ specific to classify correctly $54 \%$ of the malignant effusions [61]. Discriminating pleural fluid cut-off values were generally higher than those found in serum, a finding that does not justify the routine use of measuring classic tumour markers in the workup of pleural effusions. Despite this, one particular study has in fact demonstrated that in cases of suspicious MPE and negative cytology and in the absence of an obvious primary source that the measurement of tumour markers may be helpful as an alternate diagnostic tool [62].

The diagnosis of a mesothelioma-related pleural effusion remains difficult as few studies have reported markers with a high positive predictive value. Increased levels of pleural CA-15-3, hyaluronic acid, and spliced forms of CD44, such as exon v6 (CD44v6), have been reported; however, a discrepancy exists in the literature for CYFRA 211 to differentiate between mesothelioma and other pleural malignancies [63]. Alternatively, mesothelin, a cell surface glycoprotein that may be cleaved into the soluble mesothelin related protein (SMRP), is a moderately sensitive but highly specific marker in serum studies [64]. Studies also support the use of pleural fluid levels of mesothelin with $98 \%$ specificity and $67 \%$ sensitivity in mesothelioma compared to benign effusions $[65,66]$. Pleural SMRP measurement also diagnosed mesothelioma more reliably than cytological examination alone [65, 67]. Consequently, such a measure could be considered for patients with undiagnosed pleural effusions, particularly if mesothelioma is a concern. 
Immunocytochemistry may also be performed on cytological pleural fluid specimens. For example, several mesothelial markers such as calretinin, keratin 5/6, and WT-1 protein may be used in conjunction with carcinoma markers such as thyroid transcription factor-1 (TTF-1), CEA, and B72.3. These may be used to effectively discriminate epithelial mesothelioma from adenocarcinoma [68]. Whilst $80 \%$ of lung adenocarcinoma exhibits TTF-1 positivity, a positive TTF-1 stain in pleural fluid without an established primary cause may alternatively suggest primary non-small cell lung cancer [68]. In the setting of breast malignancy, use of pleural Her-2-neu receptor positivity has diagnostic and therapeutic implications [69]. Although useful in particular settings, the weaknesses associated with the routine use of measuring tumour biomarkers in pleural fluid should be recognized by clinicians.

4.6. Rheumatological-Related Pleural Effusions. Rheumatological-related pleural effusions usually have pleural biochemical characteristics such as protein, $\mathrm{pH}$, and glucose similar to other noninfective causes of exudates. Serum rheumatoid factor (RF) and antinuclear antibody (ANA) are more sensitive and specific for the diagnosis of rheumatoid arthritis (RA) and systematic lupus erythematosis (SLE); however, their measurement in pleural fluid does not possess the same diagnostic accuracy for disease-related pleural effusions. RF titres may be measured in pleural fluid and are often $>1: 320$ in rheumatoid pleuritis. Despite this finding, this measurement is rarely practiced in the clinical setting as the pleural levels often reflect serum values. One particular study confirmed this fact as it reported no additional diagnostic value above that of serum analysis alone [70]. Cytological assessment looking for "ragocyte" cells that consist of white blood cells with phagocytic intracellular inclusions is described in rheumatoid pleuritis but generally has low specificity. Pleural ANA is additionally not specific to SLE and may occur in malignancies. Interestingly, however, one study has suggested that pleural ANA measurement possesses good negative predictive value for SLE pleuritis and may be useful in this context [71]. Conversely, an alternative study of $n=266$ patients has shown no additional value in measuring pleural ANA in the setting of SLE pleuritis [72]. Pleural antidouble stranded DNA (dsDNA) also has good negative predictive value, whilst complement activation of pleural fluid in both RA and SLE has also been evaluated but is not routinely practiced in the clinical setting due to its low specificity [73].

\section{Conclusion}

Since the introduction of Light's criteria in the 1970s, other proposed criteria and recommendations to overcome some of its drawbacks have been suggested, however, Light's criteria have remained biochemically the most robust for differentiating exudates from transudates. This allows easier diagnosis for an underlying cause of a pleural effusion, avoiding unnecessary investigation. Nevertheless, most transudates are in fact secondary to congestive heart failure where clinical judgement and disease-specific markers such as NT-proBNP have been proven to be superior. Other useful disease-specific markers include ADA in the diagnosis of tuberculous effusions. Discriminating malignant and benign pleural effusions in the setting of an exudate remains a challenge. While there is no substitute to the histologic demonstration of malignancy, the role of tumour markers may emerge to have a larger contribution in the future as a complementary tool in the setting of MPE particularly when considering the invasiveness and lack of universal accessibility to thoracoscopy. Although workup of pleural effusions is a mix of both old and new measures, novel technologies such as global gene profiling and proteomics enable the identification of "fingerprints" for disease-specific markers that will undoubtedly improve our approach in the diagnosis for a definitive cause of a particular pleural effusion. Such future improvements do illustrate major advances from the simplistic transudate-exudate separation and do suggest that pleural fluid analysis is in fact a work in progress.

\section{References}

[1] R. W. Light, M. I. Macgregor, P. R. Luchsinger, and W. C. Ball, "Pleural effusions: the diagnostic separation of transudates and exudates," Annals of Internal Medicine, vol. 77, no. 4, pp. 507-513, 1972.

[2] S. Romero-Candeira, C. Fernández, C. Martín, J. SánchezPaya, and L. Hernández, "Influence of diuretics on the concentration of proteins and other components of pleural transudates in patients with heart failure," American Journal of Medicine, vol. 110, no. 9, pp. 681-686, 2001.

[3] J. M. Porcel, M. Vives, M. C. Vicente de Vera, G. Cao, M. Rubio, and M. C. Rivas, "Useful tests on pleural fluid that distinguish transudates from exudates," Annals of Clinical Biochemistry, vol. 38, no. 6, pp. 671-675, 2001.

[4] S. Romero-Candeira, L. Hernández, S. Romero-Brufao, D. Orts, C. Fernández, and C. Martín, "Is it meaningful to use biochemical parameters to discriminate between transudative and exudative pleural effusions?" Chest, vol. 122, no. 5, pp. 1524-1529, 2002.

[5] J. M. Porcel, J. M. Peña, C. Vicente de Vera, and A. Esquerda, "Reappraisal of the standard method (Light's criteria) for identifying pleural exudates," Medicina Clinica, vol. 126, no. 6, pp. 211-213, 2006.

[6] B. H. F. Maranhão, C. T. Silva Junior, A. M. S. Chibante, and G. P. Cardoso, "Determination of total proteins and lactate dehydrogenase for the diagnosis of pleural transudates and exudates: redefining the classical criterion with a new statistical approach," Jornal Brasileiro de Pneumologia, vol. 36, no. 4, pp. 468-474, 2010.

[7] S. Meisel, A. Shamiss, M. Thaler, N. Nussinovitch, and T. Rosenthal, "Pleural fluid to serum bilirubin concentration ratio for the separation of transudates from exudates," Chest, vol. 98, no. 1, pp. 141-144, 1990.

[8] H. Hamm, U. Brohan, R. Bohmer, and H. P. Missmahl, "Cholesterol in pleural effusions. A diagnostic aid," Chest, vol. 92, no. 2, pp. 296-302, 1987.

[9] D. Jiménez Castro, G. Díaz Nuevo, and E. Pérez-Rodríguez, "Comparative analysis of ligth's criteria and other biochemical parameters to distinguish exudates from transudates," Revista Clinica Espanola, vol. 202, no. 1, pp. 3-6, 2002.

[10] A. Fiorelli, G. Vicidomini, M. Di Domenico et al., "Vascular endothelial growth factor in pleural fluid for differential 
diagnosis of benign and malignant origin and its clinical applications," Interactive Cardiovascular and Thoracic Surgery, vol. 12, no. 3, pp. 420-424, 2011.

[11] T. Söderblom, P. Nyberg, A. M. Teppo, M. Klockars, H. Riska, and T. Pettersson, "Pleural fluid interferon- $\gamma$ and tumour necrosis factor- $\alpha$ in tuberculous and rheumatoid pleurisy," European Respiratory Journal, vol. 9, no. 8, pp. 1652-1655, 1996.

[12] M. Metintaş, Ö. Alataş, F. Alataş, Ö. Çolak, N. Özdemir, and S. Erginel, "Comparative analysis of biochemical parameters for differentiation of pleural exudates from transudates Light's criteria, cholesterol, bilirubin, albumin gradient, alkaline phosphatase, creatine kinase, and uric acid," Clinica Chimica Acta, vol. 264, no. 2, pp. 149-162, 1997.

[13] B. J. Roth, T. F. O'Meara, and W. H. Cragun, “The serumeffusion albumin gradient in the evaluation of pleural effusions," Chest, vol. 98, no. 3, pp. 546-549, 1990.

[14] H. Liao, M. J. Na, O. Dikensoy, K. B. Lane, B. Randal, and R. W. Light, "Diagnostic value of pleural fluid N-terminal probrain natriuretic peptide levels in patients with cardiovascular diseases," Respirology, vol. 13, no. 1, pp. 53-57, 2008.

[15] A. C. Long, H. R. O’Neal Jr., S. Peng, K. B. Lane, and R. W. Light, "Comparison of pleural fluid N-terminal pro-brain natriuretic peptide and brain natriuretic-32 peptide levels," Chest, vol. 137, no. 6, pp. 1369-1374, 2010.

[16] E. C. Seyhan, S. Altin, E. Cetinkaya et al., "The importance of pleural fluid and serum NT-proBNP levels in differentiating pleural effusion due to heart failure from other causes of effusion," Internal Medicine, vol. 48, no. 5, pp. 287-293, 2009.

[17] S. Janda and J. Swiston, "Diagnostic accuracy of pleural fluid NT-pro-BNP for pleural effusions of cardiac origin: a systematic review and meta-analysis," BMC Pulmonary Medicine, vol. 10, article 58, 2010.

[18] J. M. Porcel, J. Chorda, G. Cao, A. Esquerda, A. Ruiz-González, and M. Vives, "Comparing serum and pleural fluid pro-brain natriuretic peptide (NT-proBNP) levels with pleural-to-serum albumin gradient for the identification of cardiac effusions misclassified by Light's criteria," Respirology, vol. 12, no. 5, pp. 654-659, 2007.

[19] D. Shitrit, J. E. Ollech, A. Ollech et al., "Diagnostic value of complement components in pleural fluid: report of 135 cases," Respiratory Medicine, vol. 102, no. 11, pp. 1631-1635, 2008.

[20] E. A. Rakha, S. Patil, K. Abdulla, M. Abdulkader, Z. Chaudry, and I. N. Soomro, "The sensitivity of cytologic evaluation of pleural fluid in the diagnosis of malignant mesothelioma," Diagnostic Cytopathology, vol. 38, no. 12, pp. 874-879, 2010.

[21] V. Villena, A. López-Encuentra, R. García-Luján, J. EchaveSustaeta, and C. J. Á. Martínez, "Clinical implications of appearance of pleural fluid at thoracentesis," Chest, vol. 125, no. 1, pp. 156-159, 2004.

[22] J. M. Porcel and M. Vives, "Etiology and pleural fluid characteristics of large and massive effusions," Chest, vol. 124, no. 3, pp. 978-983, 2003.

[23] M. A. Smith, R. J. Battafarano, B. F. Meyers, J. B. Zoole, J. D. Cooper, and G. A. Patterson, "Prevalence of benign disease in patients undergoing resection for suspected lung cancer," Annals of Thoracic Surgery, vol. 81, no. 5, pp. 1824-1829, 2006.

[24] B. Ozcakar, C. H. Martinez, R. C. Morice et al., "Does pleural fluid appearance really matter? The relationship between fluid appearance and cytology, cell counts, and chemical laboratory measurements in pleural effusions of patients with cancer," Journal of Cardiothoracic Surgery, vol. 5, article 63, 2010.

[25] J. T. Huggins, "Chylothorax and cholesterol pleural effusion," Seminars in Respiratory and Critical Care Medicine, vol. 31, no. 6, pp. 743-750, 2010.
[26] P. P. Roberts, "Parasitic infections of the pleural space," Seminars in Respiratory Infections, vol. 3, no. 4, pp. 362-382, 1988.

[27] A. Gopi, S. M. Madhavan, S. K. Sharma, and S. A. Sahn, "Diagnosis and treatment of tuberculous pleural effusion in 2006," Chest, vol. 131, no. 3, pp. 880-889, 2007.

[28] R. Krenke, J. Nasilowski, P. Korczynski et al., "Incidence and aetiology of eosinophilic pleural effusion," European Respiratory Journal, vol. 34, no. 5, pp. 1111-1117, 2009.

[29] J. L. C. Vidriales and C. A. Antequera, "Use of pleural fluid Creactive protein in laboratory diagnosis of pleural effusions," European Journal of Medicine, vol. 1, no. 4, pp. 201-207, 1992.

[30] Ü. Y. Turay, Z. Yildirim, Y. Türköz et al., "Use of pleural fluid C-reactive protein in diagnosis of pleural effusions," Respiratory Medicine, vol. 94, no. 5, pp. 432-435, 2000.

[31] T. S. Kiropoulos, K. Kostikas, S. Oikonomidi et al., "Acute phase markers for the differentiation of infectious and malignant pleural effusions," Respiratory Medicine, vol. 101, no. 5, pp. 910-918, 2007.

[32] J. M. Porcel, C. Galindo, A. Esquerda et al., "Pleural fluid interleukin-8 and C-reactive protein for discriminating complicated non-purulent from uncomplicated parapneumonic effusions," Respirology, vol. 13, no. 1, pp. 58-62, 2008.

[33] N. Xirouchaki, N. Tzanakis, D. Bouros et al., "Diagnostic value of interleukin- $1 \alpha$, interleukin- 6 , and tumor necrosis factor in pleural effusions," Chest, vol. 121, no. 3, pp. 815-820, 2002.

[34] V. Agrenius, L. E. Gustafsson, and O. Widström, "Tumour necrosis factor- $\alpha$ and nitric oxide, determined as nitrite, in malignant pleural effusion," Respiratory Medicine, vol. 88, no. 10, pp. 743-748, 1994.

[35] M. E. San José, L. Valdes, F. J. Gonzalez-Barcala et al., "Diagnostic value of proinflammatory interleukins in parapneumonic effusions," American Journal of Clinical Pathology, vol. 133, no. 6, pp. 884-891, 2010.

[36] R. Vavetsi, S. Bonovas, P. Polizou, C. Papanastasopoulou, G. Dougekou, and N. M. Sitaras, "The diagnostic role of glycosaminoglycans in pleural effusions: a pilot study," $B M C$ Pulmonary Medicine, vol. 9, article 9, 2009.

[37] J. M. Porcel, M. Vives, G. Cao et al., "Biomarkers of infection for the differential diagnosis of pleural effusions," European Respiratory Journal, vol. 34, no. 6, pp. 1383-1389, 2009.

[38] J. M. Porcel, A. Ruiz-González, M. Falguera et al., "Contribution of a pleural antigen assay (Binax NOW) to the diagnosis of pneumococcal pneumonia," Chest, vol. 131, no. 5, pp. 14421447, 2007.

[39] Z. D. Daniil, E. Zintzaras, T. Kiropoulos et al., "Discrimination of exudative pleural effusions based on multiple biological parameters," European Respiratory Journal, vol. 30, no. 5, pp. 957-964, 2007.

[40] L. Valdés, D. Álvarez, E. San José et al., “Tuberculous pleurisy: a study of 254 patients," Archives of Internal Medicine, vol. 158, no. 18, pp. 2017-2021, 1998.

[41] J. Dinnes, J. Deeks, H. Kunst et al., "A systematic review of rapid diagnostic tests for the detection of tuberculosis infection," Health Technology Assessment, vol. 11, no. 3, pp. 1196, 2007.

[42] Q. L. Liang, H. Z. Shi, K. Wang, S. M. Qin, and X. J. Qin, "Diagnostic accuracy of adenosine deaminase in tuberculous pleurisy: a meta-analysis," Respiratory Medicine, vol. 102, no. 5, pp. 744-754, 2008.

[43] J. M. Porcel, A. Esquerda, and S. Bielsa, "Diagnostic performance of adenosine deaminase activity in pleural fluid: a single-center experience with over 2100 consecutive patients," European Journal of Internal Medicine, vol. 21, no. 5, pp. 419$423,2010$. 
[44] J. M. Porcel, “Tuberculous pleural effusion,” Lung, vol. 187, no. 5, pp. 263-270, 2009.

[45] S. Greco, E. Girardi, R. Masciangelo, G. B. Capoccetta, and C. Saltini, "Adenosine deaminase and interferon gamma measurements for the diagnosis of tuberculous pleurisy: a metaanalysis," International Journal of Tuberculosis and Lung Disease, vol. 7, no. 8, pp. 777-786, 2003.

[46] K. Dheda, R. N. van Zyl-Smit, L. A. Sechi et al., "Utility of quantitative T-cell responses versus unstimulated interferon- $\gamma$ for the diagnosis of pleural tuberculosis," European Respiratory Journal, vol. 34, no. 5, pp. 1118-1126, 2009.

[47] M. F. Baganha, A. Mota-Pinto, M. A. Pêgo, M. A. Marques, M. A. Rosa, and A. J. Cordeiro, "Neopterin in tuberculous and neoplastic pleural fluids," Lung, vol. 170, no. 3, pp. 155-161, 1992.

[48] G. Celik, A. Kaya, B. Poyraz et al., "Diagnostic value of leptin in tuberculous pleural effusions," International Journal of Clinical Practice, vol. 60, no. 11, pp. 1437-1442, 2006.

[49] L. Valdes, E. San Jose, D. Alvarez et al., "Diagnosis of tuberculous pleurisy using the biologic parameters adenosine deaminase, lysozyme, and interferon gamma," Chest, vol. 103, no. 2, pp. 458-465, 1993.

[50] K. Dheda, R. N. van-Zyl Smit, L. A. Sechi et al., "Clinical diagnostic utility of IP-10 and LAM antigen levels for the diagnosis of tuberculous pleural effusions in a high burden setting," PLoS One, vol. 4, no. 3, Article ID e4689, 2009.

[51] L. Valdés, E. S. José, J. M. A. Dobaño et al., "Diagnostic value of interleukin-12 p40 in tuberculous pleural effusions," European Respiratory Journal, vol. 33, no. 4, pp. 816-820, 2009.

[52] P. Sheen, C. M. O'Kane, K. Chaudhary et al., "High MMP9 activity characterises pleural tuberculosis correlating with granuloma formation," European Respiratory Journal, vol. 33, no. 1, pp. 134-141, 2009.

[53] M. V. Villegas, L. A. Labrada, and N. G. Saravia, "Evaluation of polymerase chain reaction, adenosine deaminase, and interferon- $\gamma$ in pleural fluid for the differential diagnosis of pleural tuberculosis," Chest, vol. 118, no. 5, pp. 1355-1364, 2000.

[54] S. M. Sallach, J. A. Sallach, E. Vasquez, L. Schultz, and P. Kvale, "Volume of pleural fluid required for diagnosis of pleural malignancy," Chest, vol. 122, no. 6, pp. 1913-1917, 2002.

[55] J. Swiderek, S. Morcos, V. Donthireddy et al., "Prospective study to determine the volume of pleural fluid required to diagnose malignancy," Chest, vol. 137, no. 1, pp. 68-73, 2010.

[56] W. Abouzgheib, T. Bartter, H. Dagher, M. Pratter, and W. Klump, "A prospective study of the volume of pleural fluid required for accurate diagnosis of malignant pleural effusion," Chest, vol. 135, no. 4, pp. 999-1001, 2009.

[57] J. Ferrer, M. A. A. Villarino, G. Encabo et al., "Diagnostic utility of CYFRA 21-1, carcinoembryonic antigen, CA 125, neuron specific enolase, and squamous cell antigen level determinations in the serum and pleural fluid of patients with pleural effusions," Cancer, vol. 86, no. 8, pp. 1488-1495, 1999.

[58] S. Bielsa, A. Esquerda, A. Salud et al., "High levels of tumor markers in pleural fluid correlate with poor survival in patients with adenocarcinomatous or squamous malignant effusions," European Journal of Internal Medicine, vol. 20, no. 4, pp. 383-386, 2009.

[59] M. Tomita, T. Shimizu, Y. Matsuzaki, M. Hara, T. Ayabe, and T. Onitsuka, "Prognostic significance of carcinoembryonic antigen level in pleural lavage fluid for patients with lung adenocarcinoma," Annals of Thoracic Surgery, vol. 80, no. 1, pp. 276-281, 2005.
[60] I. C. Wagner, M. J. Guimarães, L. K. da Silva, F. M. de Melo, and M. T. C. Muniz, "Evaluation of serum and pleural levels of the tumor markers CEA, CYFRA21-1 and CA 15-3 in patients with pleural effusion," Jornal Brasileiro de Pneumologia, vol. 33, no. 2, pp. 185-191, 2007.

[61] J. M. Porcel, M. Vives, A. Esquerda, A. Salud, B. Pérez, and F. Rodríguez-Panadero, "Use of a panel of tumor markers (carcinoembryonic antigen, cancer antigen 125, carbohydrate antigen 15-3, and cytokeratin 19 fragments) in pleural fluid for the differential diagnosis of benign and malignant effusions," Chest, vol. 126, no. 6, pp. 1757-1763, 2004.

[62] J. H. Lee and J. H. Chang, "Diagnostic utility of serum and pleural fluid carcinoembryonic antigen, neuron-specific enolase, and cytokeratin 19 fragments in patients with effusions from primary lung cancer," Chest, vol. 128, no. 4, pp. 22982303, 2005.

[63] C. Fuhrman, J. C. Duche, C. Chouaid et al., "Use of tumor markers for differential diagnosis of mesothelioma and secondary pleural malignancies," Clinical Biochemistry, vol. 33, no. 5, pp. 405-410, 2000.

[64] A. Scherpereel, B. Grigoriu, M. Conti et al., "Soluble mesothelin-related peptides in the diagnosis of malignant pleural mesothelioma," American Journal of Respiratory and Critical Care Medicine, vol. 173, no. 10, pp. 1155-1160, 2006.

[65] H. E. Davies, R. S. Sadler, S. Bielsa et al., "Clinical impact and reliability of pleural fluid mesothelin in undiagnosed pleural effusions," American Journal of Respiratory and Critical Care Medicine, vol. 180, no. 5, pp. 437-444, 2009.

[66] J. Creaney, D. Yeoman, L. K. Naumoff et al., "Soluble mesothelin in effusions: a useful tool for the diagnosis of malignant mesothelioma," Thorax, vol. 62, no. 7, pp. 569-576, 2007.

[67] A. Scherpereel, P. Astoul, P. Baas et al., "Guidelines of the European Respiratory Society and the European Society of Thoracic Surgeons for the management of malignant pleural mesothelioma," European Respiratory Journal, vol. 35, no. 3, pp. 479-495, 2010.

[68] N. Pomjanski, H. J. Grote, P. Doganay, V. Schmiemann, B. Buckstegge, and A. Böcking, "Immunocytochemical identification of carcinomas of unknown primary in serous effusions," Diagnostic Cytopathology, vol. 33, no. 5, pp. 309315, 2005.

[69] T. L. Hung, F. F. Chen, J. M. Liu et al., "Clinical evaluation of HER-2/neu protein in malignant pleural effusion-associated lung adenocarcinoma and as a tumor marker in pleural effusion diagnosis," Clinical Cancer Research, vol. 9, no. 7, pp. 2605-2612, 2003.

[70] A. Balbir-Gurman, M. Yigla, A. M. Nahir, and Y. BraunMoscovici, "Rheumatoid pleural effusion," Seminars in Arthritis and Rheumatism, vol. 35, no. 6, pp. 368-378, 2006.

[71] D. Y. Wang, P. C. Yang, W. L. Yu, S. H. Kuo, and N. Y. Hsu, "Serial anitnuclear antibodies titre in pleural and pericardial fluid," European Respiratory Journal, vol. 15, no. 6, pp. 11061110, 2000.

[72] J. M. Porcel, J. Ordi-Ros, A. Esquerda et al., "Antinuclear antibody testing in pleural fluid for the diagnosis of lupus pleuritis," Lupus, vol. 16, no. 1, pp. 25-27, 2007.

[73] E. R. Salomaa, M. Viander, T. Saaresranta, and E. O. Terho, "Complement components and their activation products in pleural fluid," Chest, vol. 114, no. 3, pp. 723-730, 1998. 


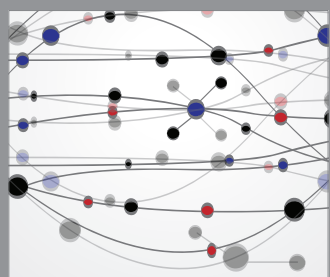

The Scientific World Journal
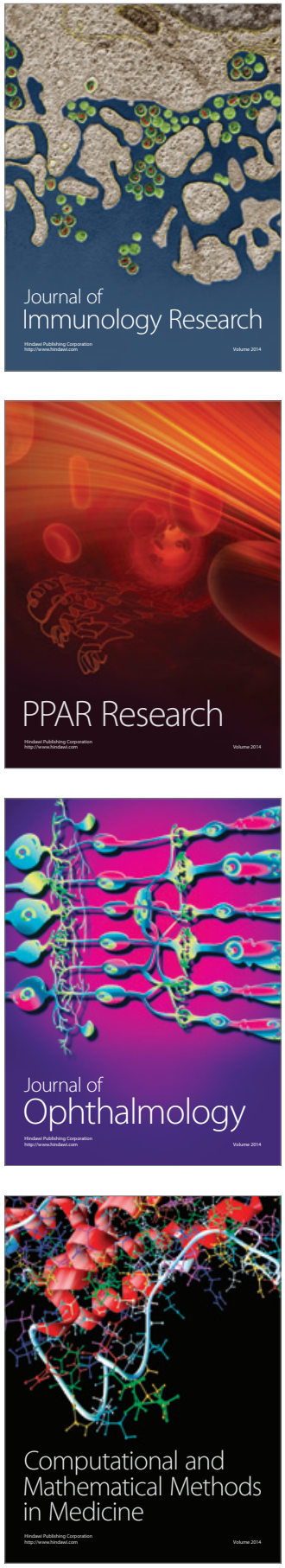

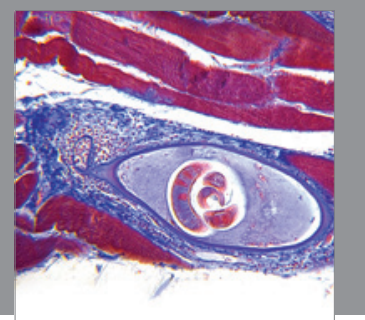

Gastroenterology

Research and Practice
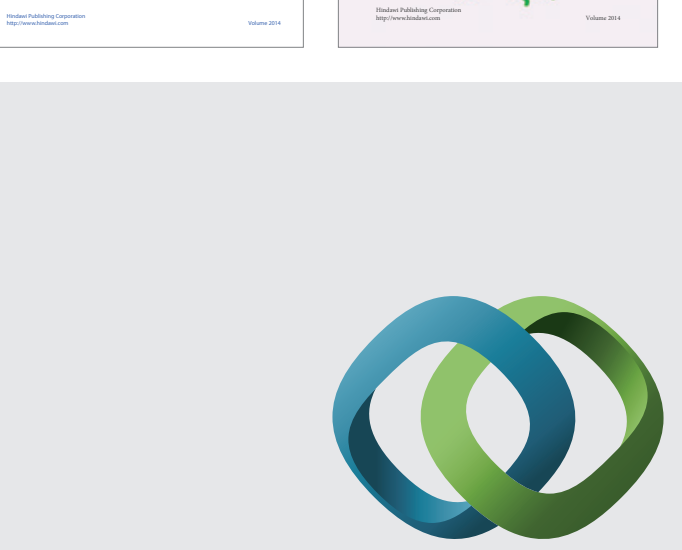

\section{Hindawi}

Submit your manuscripts at

http://www.hindawi.com
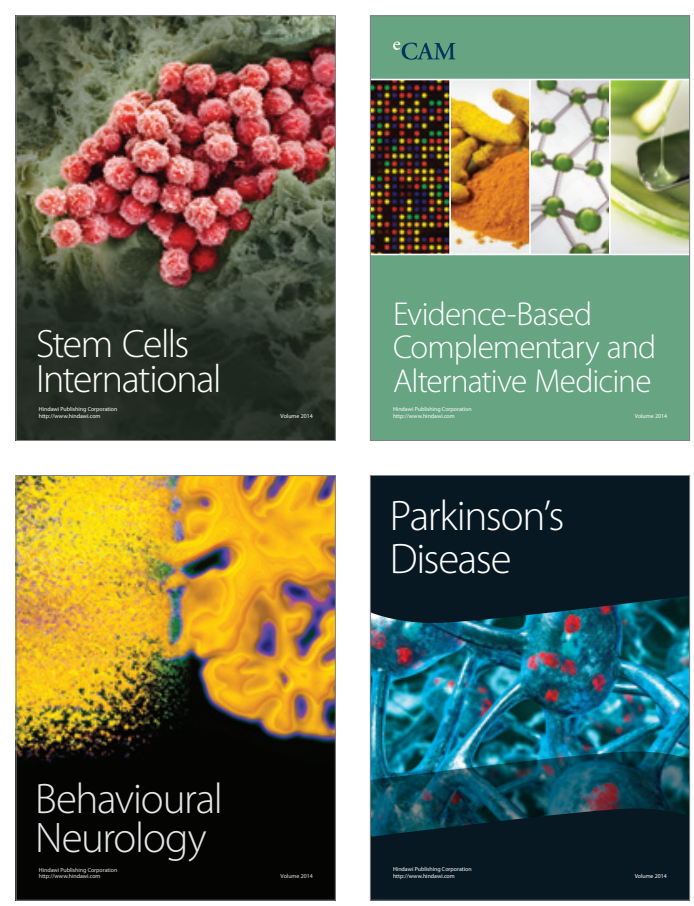

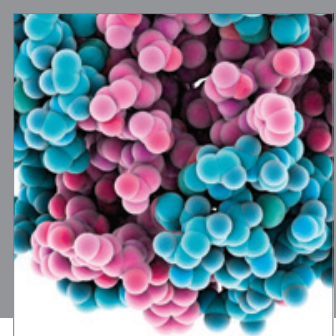

Journal of
Diabetes Research

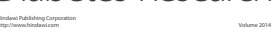

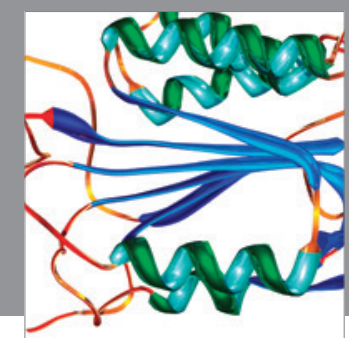

Disease Markers
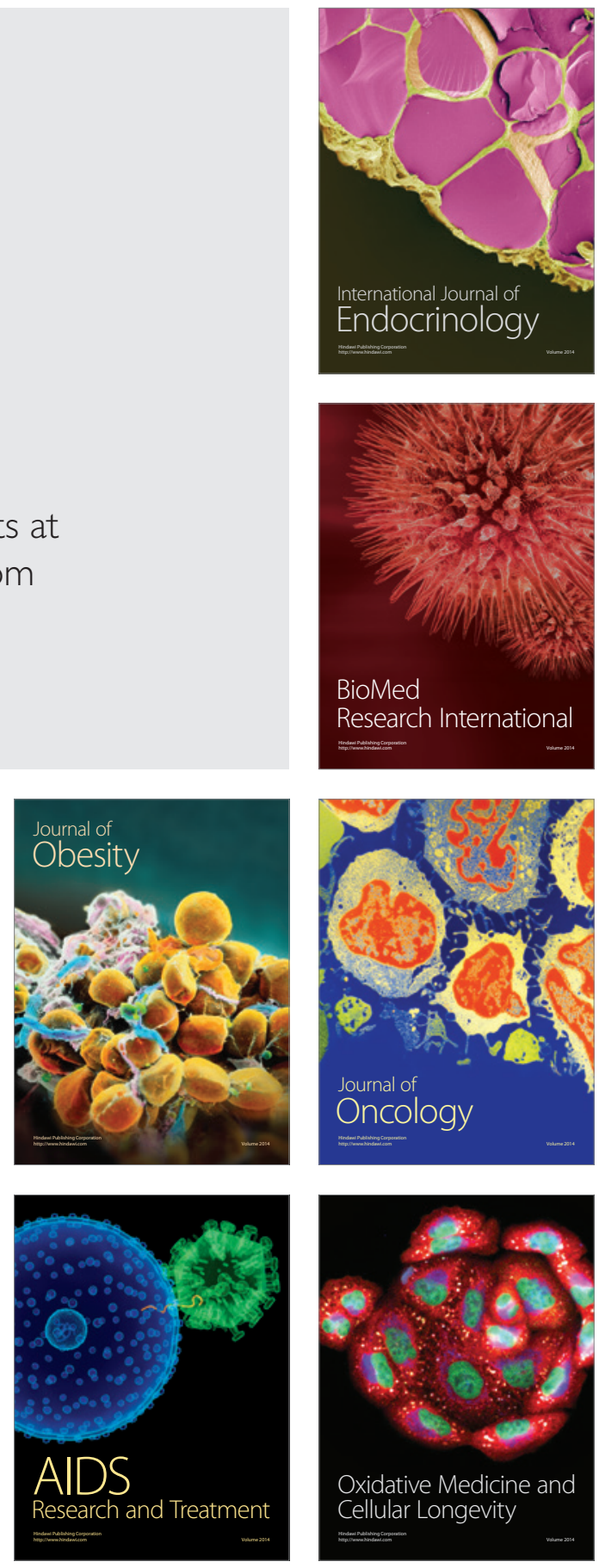